Supporting Information

\title{
Mechanism and Application of a Microcapsule Enabled Multicatalyst Reaction
}

\author{
Sarah L. Poe, Muris Kobašlija, D. Tyler McQuade* \\ Department of Chemistry and Chemical Biology, Baker Laboratory, Cornell University, \\ Ithaca, New York 14853-1301. \\ *Corresponding author e-mail: $\underline{\mathrm{dtm} 25 @ \text { cornell.edu }}$
}

\section{Materials and instrumentation}

Celite 545 (Acros), 4-cyanobenzaldehyde (Acros, 98\%), isobutyraldehyde (Acros, 99+\%), isovaleraldehyde (Acros, 98\%), 4-nitrobenzaldehyde (Acros, 99\%), $p$-tolualdehyde (Acros, 99+\%), trifluoroacetic anhyhdride (Acros, 99+\%), valeraldehyde (Acros, 98\%), ( \pm )-trans-1,2diaminocyclohexane (Aldrich, 98\%), mesitylene (Aldrich, 98\%), trans- $\beta$-nitrostyrene (Aldrich, 99\%), polyisobutylene (Aldrich, MW 400, 000), Raney Ni (Aldrich, pH 10, 50\% solution in water), tolylene 2,4-diisocyanate (Aldrich, technical grade, 80\%), (1S,2S)-(+)-1,2diaminocyclohexane (Alfa Aesar, 98\%), chloroform (J. T. Baker), hydrochloric acid (J. T. Baker, 36.5-38\%), nitromethane (J. T. Baker, 99\%), acetic anhydride (Mallinckrodt), cyclohexane (Mallinckrodt), methanol (Mallinckrodt), toluene (Mallinckrodt), ethanol (Pharmaco-AAPER, 200 proof), branched poly(ethyleneimine) (Polysciences, Inc., 10,000 MW), $p$-anisaldehyde (Sigma), and Span 85 (Sigma) were used as received. Dimethyl malonate (Acros, 97\%) and benzaldehyde (Aldrich, 99.5\%) were washed with saturated $\mathrm{NaHCO}_{3}$, distilled, and dried over $\mathrm{Na}_{2} \mathrm{SO}_{4}$ prior to use. 4-Bromobenzaldehyde (Aldrich, 99\%) and 4-chlorobenzaldehyde (Aldrich, 97\%) were recrystallized from $95 \% \mathrm{EtOH}$ prior to use.

Reactions were rocked on a Thermolyne Speci-Mix test tube rocker.

Hydrogenations were performed in a PARR hydrogenation apparatus 3911EA.

For ${ }^{19} \mathrm{~F}$ elemental analysis, $\mu$ caps acylated with trifluoroacetic anhydride were sent to Robertson Microlit Laboratories for analysis (www.robertson-microlit.com).

For ${ }^{16} \mathrm{O}$ elemental analysis, $\mu$ caps were sent to Elemental Analysis, Inc. for analysis (elementalanalysis.com).

Gas chromatographic (GC) analyses were performed using a Varian CP-3800 GC equipped with a Varian CP-8400 autosampler, a flame ionization detector (FID) and a Varian CP-Sil 5CB column (length $=15 \mathrm{~m}$, inner diameter $=0.25 \mathrm{~mm}$, and film thickness $=0.25 \mu \mathrm{m}$. The temperature program for $\mathrm{GC}$ analysis held the temperature constant at $80^{\circ} \mathrm{C}$, heated samples from 80 to $200{ }^{\circ} \mathrm{C}$ at $17{ }^{\circ} \mathrm{C} / \mathrm{min}$, and held at $200{ }^{\circ} \mathrm{C}$ for $2 \mathrm{~min}$. Inlet and detector temperatures were set constant at 220 and $250^{\circ} \mathrm{C}$, respectively. Mesitylene was used as an internal standard to calculate reaction conversion. 
High Performance Liquid Chromatography (HPLC) analyses were performed using a HewlettPackard Series II 1090 Liquid Chromatograph equipped with an autosampler HP 79846A, a solvent delivery system HP 79835A and a diode-array detector HP 79883A.

${ }^{1} \mathrm{H}$ NMR and ${ }^{13} \mathrm{C}$ NMR spectra were recorded on a Varian Inova-400 (400 MHz) spectrometer and are reported in ppm using solvent as an internal standard. Data are reported as $\mathrm{s}=$ singlet, $\mathrm{d}$ $=$ doublet, $\mathrm{t}=$ triplet, quin $=$ quintet, $\mathrm{m}=$ multiplet .

Optical microscopy images were obtained on an inverted Leica DMIL with a mounted Sony DSC-F717 digital camera and ebq100 UV source.

\section{Catalyst synthesis}

\subsection{Synthesis of microencapsulated poly(ethyleneimine) catalyst}

The microcapsule catalyst was prepared by interfacial polymerization of oil-in-oil emulsions, in a slightly different manner than what was described by Kobašlija and McQuade. ${ }^{[1]}$ To cyclohexane $(50 \mathrm{~mL}, \eta=9.5 \mathrm{cp})$ and Span 85 mixture $(2 \% \mathrm{v} / \mathrm{v})$ stirred at $1500 \mathrm{rpm}$ with a magnetic stirrer, the disperse phase (1.5 mL PEI in $6.0 \mathrm{~mL}$ methanol and $1.5 \mathrm{~mL}$ chloroform) was added at once. After 2 minutes of stirring, 2,4-tolylene diisocyanate (TDI, $1.0 \mathrm{~mL}$ in $9.0 \mathrm{~mL}$ cyclohexane) was added at once and the stirring was reduced to $500 \mathrm{rpm}$. After 1 minute, polymerization was stopped by the addition of cyclohexane $(30 \mathrm{~mL})$. The resulting capsules were left to settle, further washed with hexanes, and left to air-dry overnight.

\subsection{Catalyst loading}

Primary amine loading for the microcapsule catalyst was determined via labeling with fluorine and a subsequent fluorine elemental analysis. To microcapsule catalyst $(100 \mathrm{mg})$, loaded in a syringe equipped with a frit, methanol $(5 \mathrm{~mL})$ was added to swell them. After 5 minutes the excess methanol was removed and the solution containing trifluoroacetic anhydride $(1 \mathrm{~mL})$ in methanol $(5 \mathrm{~mL})$ was drawn into the syringe. The mixture was rocked at room temperature overnight. Fluorine labeled microcapsules were extensively washed with methanol, dried under stream of $\mathrm{N}_{2}$ and sent for fluorine elemental analysis. To ensure that all the active sites were acylated, the microcapsules were checked for the activity in nitroalkene formation. As expected for fully acylated microcapsules, showed no activity. Elemental analysis revealed a fluorine loading of 26.54 mass \%.

$$
\frac{0.2654 \mathrm{~g} \mathrm{~F}}{1 \mathrm{~g} \mathrm{\mu \text {caps }}} \cdot \frac{1 \mathrm{~mol} \mathrm{~F}}{19 \mathrm{~g} \mathrm{~F}} \cdot \frac{1 \mathrm{~mol} \mathrm{"N"}}{3 \mathrm{~mol} \mathrm{~F}} \cdot \frac{1000 \mathrm{mmol} \text { "N" }}{1 \mathrm{~mol} \mathrm{"N"}}=4.66 \mathrm{mmol} \text { "N"/g }
$$

where "N" is any amine that is capable of being trifluoroacylated $\left(1^{\circ}\right.$ and $2^{\circ}$ amines). Since the primary, secondary, and tertiary amines of PEI statistically exist in a 1:2:1 ratio, the loading of $1^{\circ}$ amines in the microencapsulated catalyst is approximately $1.55 \mathrm{mmol} / \mathrm{g}$. 
The $1^{\circ}$ amine loading of $10,000 \mathrm{MW}$ PEI was calculated based on the assumption that the primary, secondary, and tertiary amines exist in a 1:2:1 ratio, as stated by the vendor. The $1^{\circ}$ amine loading was calculated to be $5.81 \mathrm{mmol} / \mathrm{g}$.

The urea loading of the microencapsulated catalyst was determined by oxygen elemental analysis after the $\mu$ caps were dried under vacuum. Results indicated that the oxygen loading was 9.03 mass $\%$.

$$
\frac{0.0903 \mathrm{~g} \mathrm{O}}{1 \mathrm{~g} \mathrm{\mu caps}} \cdot \frac{1 \mathrm{~mol} \mathrm{O}}{16 \mathrm{~g} \mathrm{O}} \cdot \frac{1000 \mathrm{mmol} \mathrm{O}}{1 \mathrm{~mol} \mathrm{O}}=5.64 \mathrm{mmol} \mathrm{O} / \mathrm{g}
$$

The oxygen content of the acylated $\mu$ caps (see Section 3.4) was found to be 15.83 mass $\%$.

$$
\frac{0.1583 \mathrm{~g} \mathrm{O}}{1 \mathrm{~g} \mu \text { caps }} \cdot \frac{1 \mathrm{~mol} \mathrm{O}}{16 \mathrm{~g} \mathrm{O}} \cdot \frac{1000 \mathrm{mmol} \mathrm{O}}{1 \mathrm{~mol} \mathrm{O}}=9.89 \mathrm{mmol} \mathrm{O} / \mathrm{g}
$$

Assuming that $5.64 \mathrm{mmol} \mathrm{O/g}$ is due to urea groups, there are $4.26 \mathrm{mmol} \mathrm{O} / \mathrm{g}$ due to the acylation of $1^{\circ}$ and $2^{\circ}$ amines, consistent with the findings above.

\subsection{Synthesis of nickel-based Michael addition catalyst}

The catalyst was prepared in accordance with the preparation described by Seidel and Evans using $( \pm)$-trans-1,2-diaminocyclohexane or $(1 S-2 S)-(+)$-diaminocyclohexane. ${ }^{[2]}$

\section{Catalytic studies}

\subsection{Mcap-catalyzed reaction in different solvents}

Either free PEI (7.9 mg, 4.6 mole $\% 1^{\circ}$ amines) or encapsulated PEI (1, $30 \mathrm{mg}, 4.6$ mole $\% 1^{\circ}$ amines) was placed in a $4 \mathrm{~mL}$ glass vessel. Solvent $(1 \mathrm{~mL})$ was added, and the vessel was sealed and allowed to stand at $297 \mathrm{~K}$ overnight. For the 1:3 ethanol:toluene mixture, the caps were swollen in $0.25 \mathrm{~mL}$ ethanol for 10 minutes before the addition of $0.75 \mathrm{~mL}$ toluene. Benzaldehyde (4, $101.6 \mu \mathrm{L}, 1 \mathrm{mmol})$, nitromethane $(0.54 \mathrm{~mL}, 10 \mathrm{mmol})$, and mesitylene (13.9 $\mu \mathrm{L}, 0.1 \mathrm{mmol}$ ) were added. The vessel was sealed and the reaction was rocked at $297 \mathrm{~K}$ on a rocker. Reaction conversion was monitored by withdrawing aliquots from the reaction at different time intervals, diluting with methylene chloride, and analyzing by GC with reference to mesitylene. 


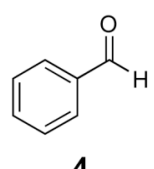

4

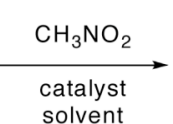

solvent

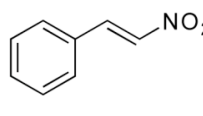

5

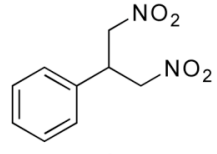

6

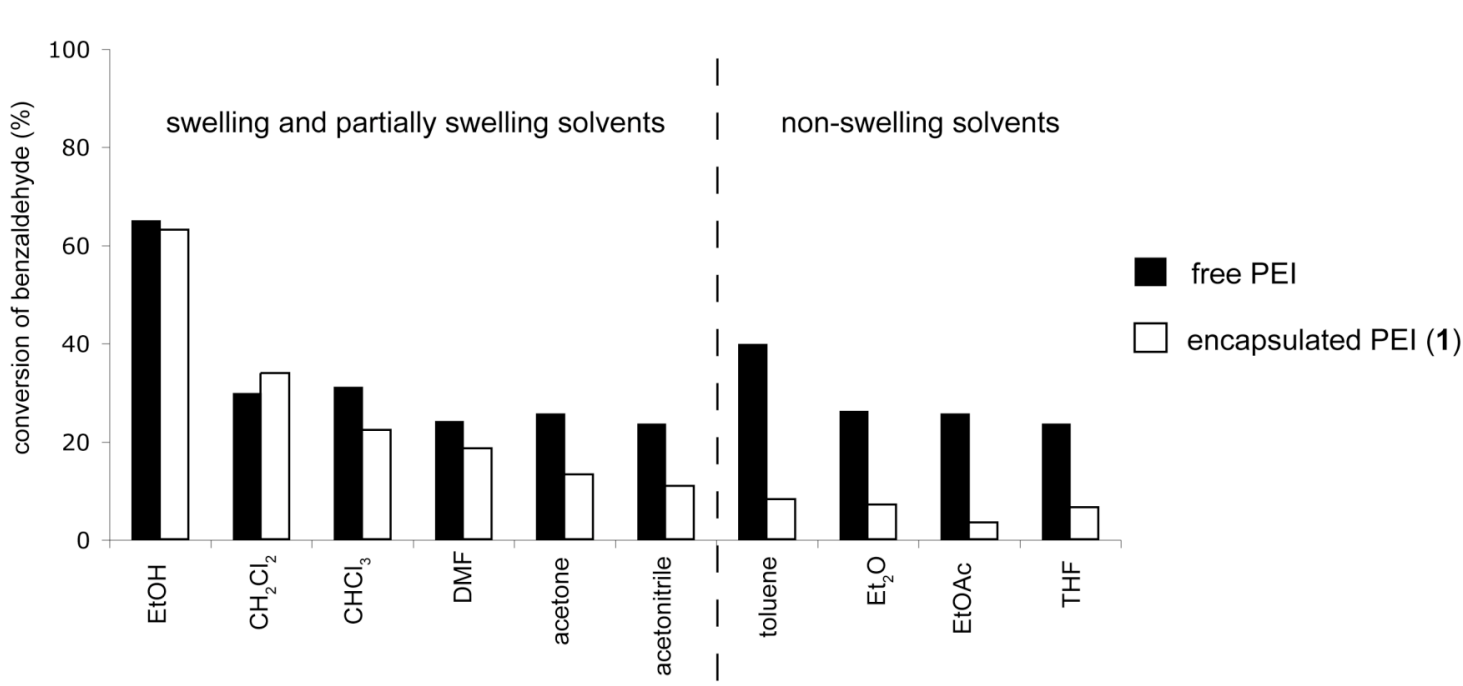

Figure S1. Conversion of benzaldehyde (4) after 6 hours for the amine-catalyzed reaction between benzaldehyde and nitromethane. Catalysts for the reaction were free PEI (black, 4.6 mol \%) and encapsulated PEI (white, $4.6 \mathrm{~mol} \%$ ).

trans- $\beta$-Nitrostyrene (5). The title compound can be commercially obtained from Aldrich for comparison purposes. ${ }^{1} \mathrm{H}$ NMR $\left(400 \mathrm{MHz}, \mathrm{CDCl}_{3}\right) \delta 7.90(\mathrm{~d}, J=13.65 \mathrm{~Hz}, 1 \mathrm{H}), 7.53(\mathrm{~d}, J=$ 13.65 Hz, 1H), 7.48-7.35 (m, 5H); ${ }^{13} \mathrm{C}$ NMR (400 MHz, $\left.\mathrm{CDCl}_{3}\right) \delta$ 139.1, 137.1, 132.2, 130.1, 129.4, 129.3 .

1,3-dinitro-2-phenyl-propane (6). The title compound can be purified by column chromatography (20\%EtOAc/hexanes) to give a brown oil. ${ }^{1} \mathrm{H}$ NMR $\left(400 \mathrm{MHz}, \mathrm{CDCl}_{3}\right) \delta$ 7.36$7.29(\mathrm{~m}, 3 \mathrm{H}), 7.2-7.17(\mathrm{~m}, 2 \mathrm{H}), 4.76-4.65(\mathrm{~m}, 4 \mathrm{H}), 4.3-4.22$ (quin, $J=7.21 \mathrm{~Hz}, 1 \mathrm{H}) ;{ }^{13} \mathrm{C} \mathrm{NMR}$ $\left(400 \mathrm{MHz}, \mathrm{CDCl}_{3}\right) \delta$ 134.5, 129.7, 129.2, 127.6, 76.9, 41.9.

\subsection{Evidence against nitroalcohol intermediate}

2-nitro-1-phenylethanol. The title compound was prepared according to the following procedure: Benzaldehyde $(4,0.48 \mathrm{~mL}, 4.7 \mathrm{mmol})$, nitromethane $(0.5 \mathrm{~mL}, 9.2 \mathrm{mmol})$, triethylamine (6 drops), and $\mathrm{EtOH}(0.54 \mathrm{~mL})$ were stirred at room temperature for 18 hours. The volatile components were removed under reduced pressure and the crude product was purified by column chromatography (20\% EtOAc/hexanes). ${ }^{1} \mathrm{HNMR}\left(400 \mathrm{MHz}, \mathrm{CDCl}_{3}\right) \delta$ 7.46-7.41 (m, $5 \mathrm{H}), 5.52-5.48(\mathrm{dd}, 1 \mathrm{H}), 4.66-4.62(\mathrm{dd}, 1 \mathrm{H}), 4.58-4.53(\mathrm{dd}, 1 \mathrm{H}), 3.86(\mathrm{~s}, 1 \mathrm{H}) ;{ }^{13} \mathrm{CNMR}(400$ $\left.\mathrm{MHz}, \mathrm{CDCl}_{3}\right) \delta 138.4,129.2,129.1,126.2,81.4,71.2$. 
Microencapsulated PEI catalyst (1, $25 \mathrm{mg}, 11.5 \mathrm{~mol} \%)$ was swollen in $1 \mathrm{~mL}$ methanol in a 4 $\mathrm{mL}$ glass vessel before use. 2-nitro-1-phenylethanol (167 mg, $1 \mathrm{mmol})$ and mesitylene were added to the vessel, which was sealed and rocked at room temperature on a rocker. Reaction conversion was monitored by withdrawing aliquots from the reaction at different time intervals, diluting with methylene chloride, and analyzing by GC with reference to mesitylene. No trans$\beta$-nitrostyrene (5) formation was observed over the course of the reaction.

\subsection{Tandem reaction}

General procedure: Microencapsulated PEI catalyst (1, $15 \mathrm{mg}, 6.9 \mathrm{~mol} \%)$ was swollen in 0.5 $\mathrm{mL}$ methanol in a $4 \mathrm{~mL}$ glass vessel before use. Nickel catalyst (2, $60 \mathrm{mg}, 7.4 \mathrm{~mol} \%$ ), aldehyde ( $1 \mathrm{mmol})$, nitromethane $(0.54 \mathrm{~mL}, 10 \mathrm{mmol})$, dimethyl malonate $(114.3 \mu \mathrm{L}, 1 \mathrm{mmol})$, and toluene $(1 \mathrm{~mL})$ were added to the vessel, which was sealed with a screw cap. The reaction was rocked at room temperature on a rocker for 24 hours. The volatile components were removed under reduced pressure and the crude product was purified by column chromatography.

Methyl-2-carbomethoxy-4-nitro-3-phenyl-butyrate (7a). The title compound can be purified by column chromatography $\left(20 \%\right.$ EtOAc/hexanes) to give a white solid. ${ }^{1} \mathrm{H}$ NMR $(400 \mathrm{MHz}$, $\left.\mathrm{CDCl}_{3}\right) \delta$ 7.33-7.26 (m, 3H), 7.24-7.21 (m, 3H), 4.95-4.84 (m, 2H), $4.24(\mathrm{dt}, J=5.1 \mathrm{~Hz}, 9.0 \mathrm{~Hz}$, $1 \mathrm{H}), 3.86(\mathrm{~d}, J=9.16,1 \mathrm{H}), 3.75(\mathrm{~s}, 1 \mathrm{H}), 3.54(\mathrm{~s}, 1 \mathrm{H}) ;{ }^{13} \mathrm{C} \mathrm{NMR}\left(400 \mathrm{MHz}, \mathrm{CDCl}_{3}\right) \delta 168.1$, $167.5,136.4,129.3,128.7,128.1,77.6,54.9,53.3,53.1,43.2$.

Methyl-2-carbomethoxy-4-nitro-3-(4-methylphenyl)butyrate (7b). The title compound can be purified by column chromatography $\left(20 \%\right.$ EtOAc/hexanes) to give a white solid. ${ }^{1} \mathrm{HNMR}$ $\left(400 \mathrm{MHz}, \mathrm{CDCl}_{3}\right) \delta$ 7.16-7.10 (m, 4H), 4.93-4.86 (dd, 2H), 4.24-4.18 (m, 1H), 3.87-3.84 (d, $1 \mathrm{H}), 3.79(\mathrm{~s}, 3 \mathrm{H}), 3.60(\mathrm{~s}, 3 \mathrm{H}), 2.33(\mathrm{~s}, 3 \mathrm{H}) ;{ }^{13} \mathrm{CNMR}\left(400 \mathrm{MHz}, \mathrm{CDCl}_{3}\right) \delta 168.1,167.5,138.3$, $133.2,129.9,127.9,77.7,55.0,53.2,53.2,53.0,42.8,21.3$.

Methyl-2-carbomethoxy-4-nitro-3-(4-methoxyphenyl)butyrate (7c). The title compound can be purified by column chromatography $(20 \%$ EtOAc/hexanes $)$ to give a white solid. ${ }^{1} \mathrm{HNMR}$ $\left(400 \mathrm{MHz}, \mathrm{CDCl}_{3}\right) \delta$ 7.18-7.15 (d, 2H), 6.83-6.80 (d, 2H), 4.90-4.83 (dd, 2H), 4.23-4.17 (m, $1 \mathrm{H}), 3.83-3.81(\mathrm{~d}, 1 \mathrm{H}), 3.80(\mathrm{~s}, 3 \mathrm{H}), 3.79(\mathrm{~s}, 3 \mathrm{H}), 3.59(\mathrm{~s}, 3 \mathrm{H}) ;{ }^{13} \mathrm{CNMR}\left(400 \mathrm{MHz}, \mathrm{CDCl}_{3}\right) \delta$ $168.1,167.5,159.7,129.2,128.1,114.6,77.9,55.4,55.1,53.2,53.1,42.5,12.1$.

Methyl-2-carbomethoxy-4-nitro-3-(4-bromophenyl)butyrate (7d). The title compound can be purified by column chromatography (20\% EtOAc/hexanes) to give a colorless oil. ${ }^{1} \mathrm{HNMR}(400$ $\left.\mathrm{MHz}, \mathrm{CDCl}_{3}\right) \delta$ 7.48-7.44 (d, 2H), 7.16-7.12 (d, 2H), 4.97-4.82 (m, 2H), 4.28-4.20 (dt, 1H), 3.86-3.82 (d, $1 \mathrm{H}), 3.78(\mathrm{~s}, 3 \mathrm{H}), 3.60(\mathrm{~s}, 3 \mathrm{H}) ;{ }^{13} \mathrm{CNMR}\left(400 \mathrm{MHz}, \mathrm{CDCl}_{3}\right) \delta 167.8,167.2,135.4$, $132.4,129.8,122.7,77.3,54.6,53.3,53.2,42.6$.

Methyl-2-carbomethoxy-4-nitro-3-(4-chlorophenyl)butyrate (7e). The title compound can be purified by column chromatography $\left(20 \%\right.$ EtOAc/hexanes) to give a colorless oil. ${ }^{1} \mathrm{HNMR}(400$ $\left.\mathrm{MHz}, \mathrm{CDCl}_{3}\right) \delta$ 7.36-7.32 (d, 2H), 7.20-7.16 (d, 2H), 4.96-4.88 (m, 2H), 4.26-4.20 (m, 1H), 3.83-3.80 (d, 1H), $3.77(\mathrm{~s}, 3 \mathrm{H}), 3.60(\mathrm{~s}, 3 \mathrm{H}) ;{ }^{13} \mathrm{CNMR}\left(400 \mathrm{MHz}, \mathrm{CDCl}_{3}\right) \delta 167.8,167.3,134.9$, $143.5,129.6,129.4,77.4,54.6,53.3,53.1,42.5$. 
Methyl-2-carbomethoxy-3-(nitromethyl)-4-methylpentanoate (7h). The title compound can be purified by column chromatography $\left(20 \%\right.$ EtOAc/hexanes) to give a colorless oil. ${ }^{1} \mathrm{HNMR}$ $\left(400 \mathrm{MHz}, \mathrm{CDCl}_{3}\right) \delta$ 4.78-4.72 (dd, $\left.1 \mathrm{H}\right), 4.62-4.57(\mathrm{dd}, 1 \mathrm{~h}), 3.79$ (s, 3H), 3.76 (s, 3H), 3.74-3.70 $(\mathrm{d}, 1 \mathrm{H}), 2.97-2.94(\mathrm{~m}, 1 \mathrm{H}), 1.90-1.91(\mathrm{~m}, 1 \mathrm{H}), 0.98-0.94(\mathrm{~m}, 6 \mathrm{H}) ;{ }^{13} \mathrm{CNMR}\left(400 \mathrm{MHz}, \mathrm{CDCl}_{3}\right) \delta$ $169.0,168.8,75.6,53.2,53.0,51.5,42.8,29.8,20.0,19.4$.

Methyl-2-carbomethoxy-3-(nitromethyl)-5-methylhexanoate (7i). The title compound can be purified by column chromatography (20\% EtOAc/hexanes) to give a colorless oil. ${ }^{1} \mathrm{HNMR}(400$ $\left.\mathrm{MHz}, \mathrm{CDCl}_{3}\right)$ 4.70-4.63 (dd, 1H), 4.53-4.46 (dd, 1H), $3.77(\mathrm{~s}, 3 \mathrm{H}), 3.76(\mathrm{~s}, 3 \mathrm{H}), 3.63-3.61(\mathrm{~d}$, 1H), 2.98-2.92 (m, 1H), 1.67-1.61 (m, 1H), 1.31-1.26 (m, 2H), 0.91-0.87 (m, 6H); ${ }^{13} \mathrm{CNMR}(400$ $\left.\mathrm{MHz}, \mathrm{CDCl}_{3}\right) \delta 168.6,168.4,76.9,53.1,52.9,52.5,39.1,35.1,25.3,22.6,22.4$. 


\subsection{Kinetic studies for tandem reaction}

Microencapsulated PEI catalyst (1, $25 \mathrm{mg}, 3.83 \mathrm{~mol} \%$ ) was swollen in $0.5 \mathrm{~mL}$ methanol in a $4 \mathrm{~mL}$ glass vessel before use. Nickel catalyst 2 , aldehyde $8(108.2 \mu \mathrm{L}, 1 \mathrm{mmol})$, nitromethane $(0.54 \mathrm{~mL}, 10 \mathrm{mmol})$, dimethyl malonate $(114.3 \mu \mathrm{L}, 1 \mathrm{mmol})$, and toluene $(1 \mathrm{~mL})$ were added to the vessel, which was sealed with a screw cap. The reaction was rocked at room temperature on a rocker. Reaction conversion was monitored by withdrawing aliquots from the reaction at different time intervals, diluting with methylene chloride, and analyzing by $\mathrm{GC}$ with reference to mesitylene.

Data Summary:

\begin{tabular}{cccc}
\hline mg Ni cat & mole \% Ni cat & rate $(\mu \mathrm{mol} \mathbf{7 i} / \mathrm{min})$ & std dev \\
\hline 27.5 & 3.4 & 0.305 & 0.037 \\
55 & 6.8 & 0.520 & 0.031 \\
85 & 10.5 & 0.689 & 0.035 \\
110 & 13.6 & 0.915 & 0.029 \\
165 & 20.4 & 1.714 & 0.143 \\
220 & 27.2 & 1.952 & 0.190 \\
\hline
\end{tabular}
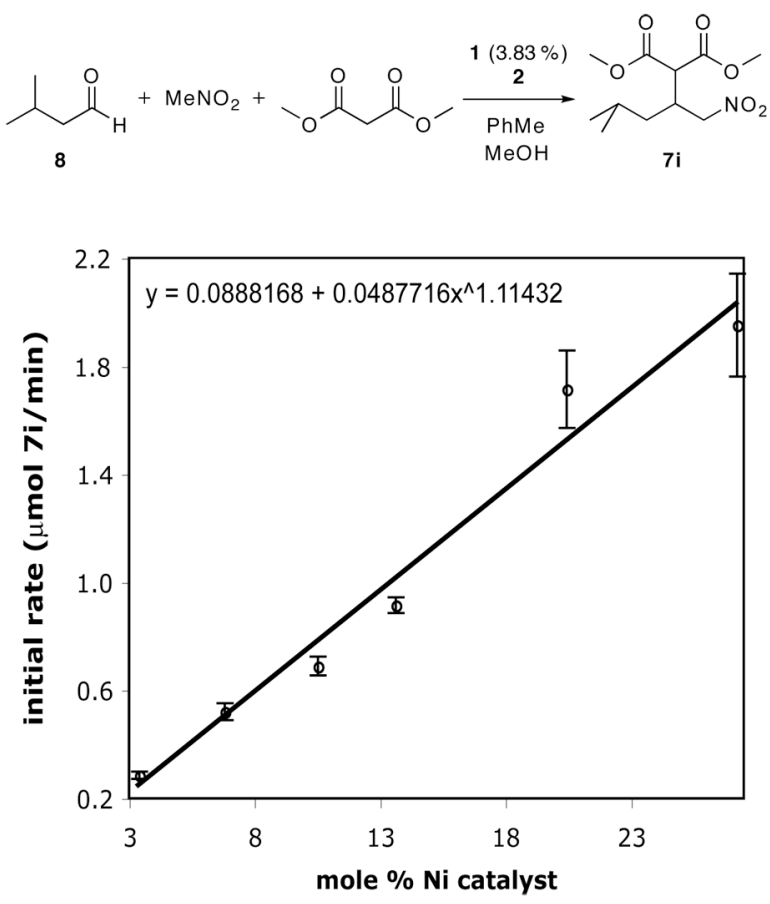


\section{Initial Rate Data}

$27.5 \mathrm{mg}$ Ni catalyst

Rate $=0.0305$

Std Dev $=0.0037$

27.5 mg Ni catalyst, run 1

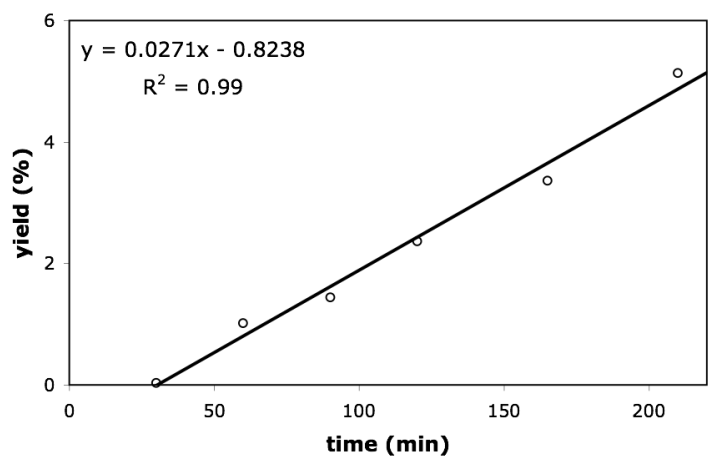

$27.5 \mathrm{mg}$ Ni catalyst, run 2

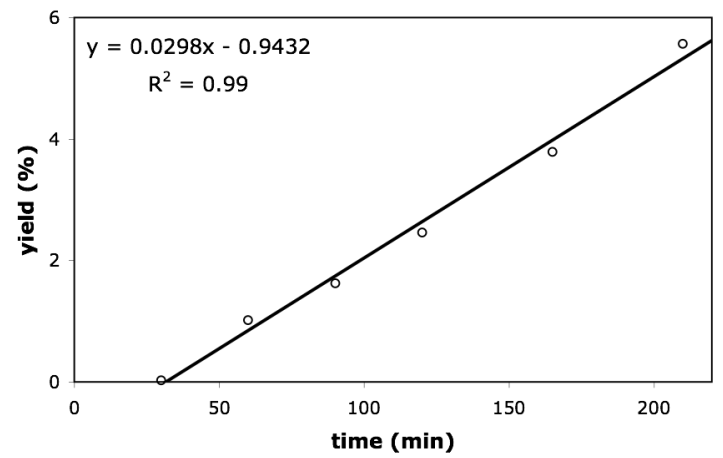

27.5 mg Ni catalyst, run 3

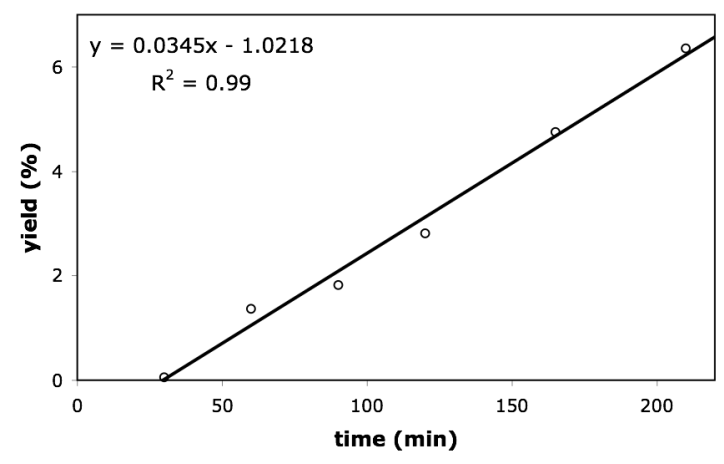


$55 \mathrm{mg}$ Ni catalyst

Rate $=0.0520$

Std Dev $=0.0031$

55 mg Ni catalyst, run 1

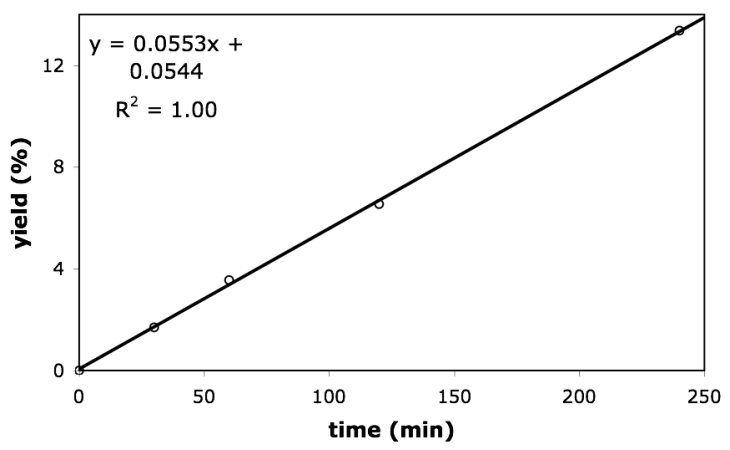

55 mg Ni catalyst, run 2

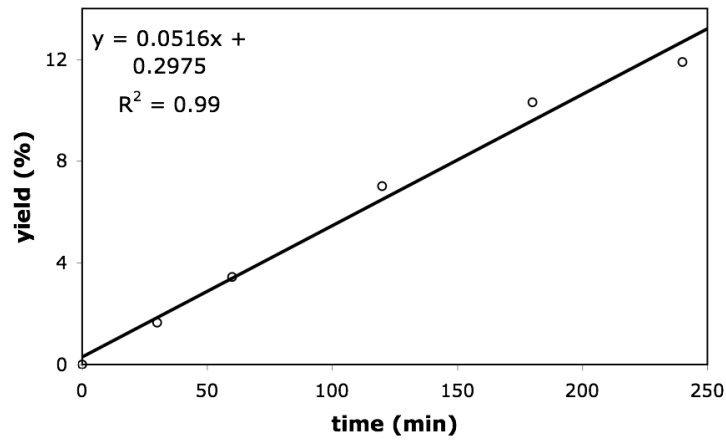

55 mg Ni catalyst, run 3

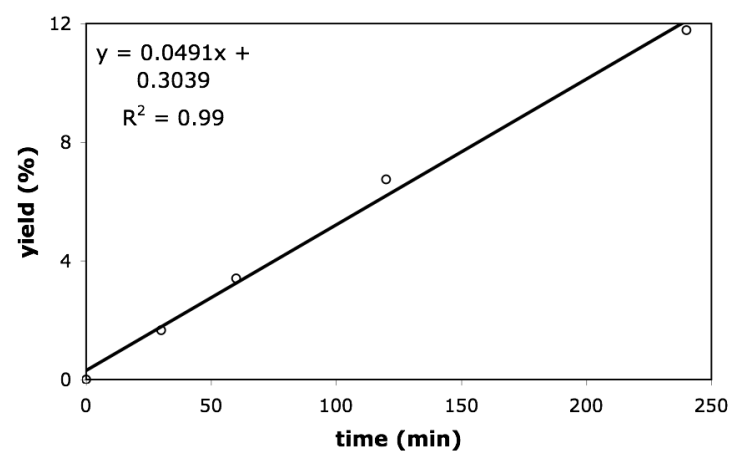


$85 \mathrm{mg}$ Ni catalyst

Rate $=0.0689$

Std Dev $=0.0035$

85 mg Ni catalyst, run 1

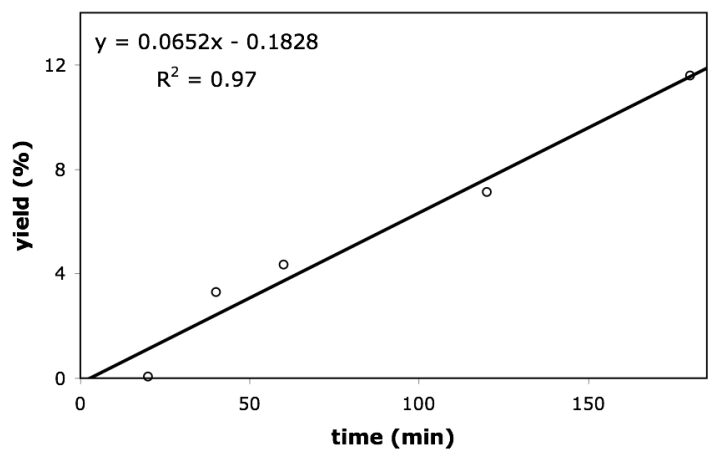

85 mg Ni catalyst, run 2

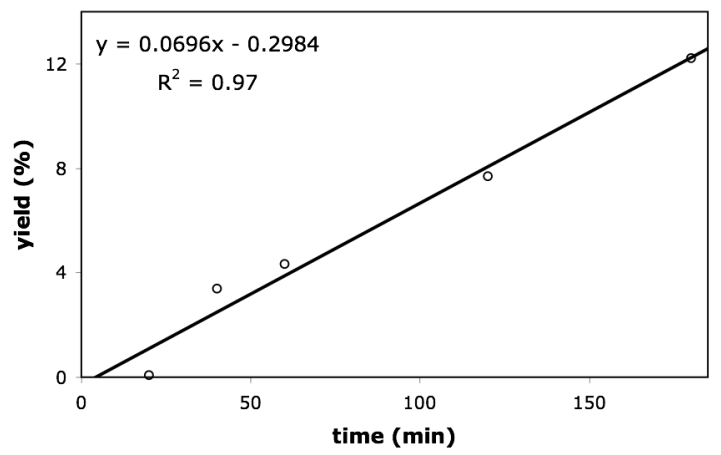

85 mg Ni catalyst, run 3

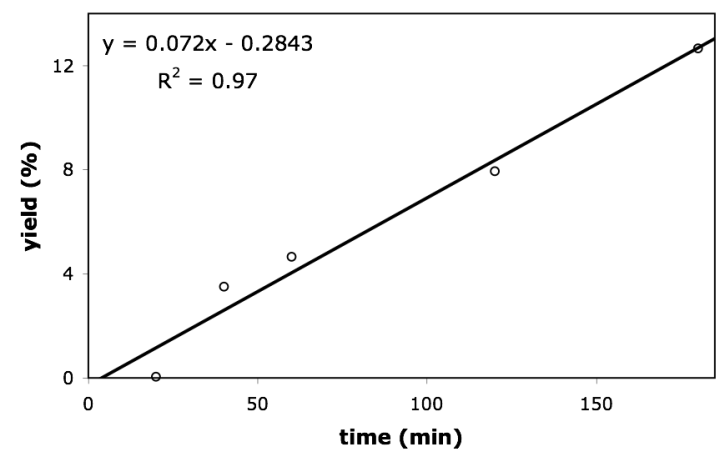


$110 \mathrm{mg}$ Ni catalyst

Rate $=0.0915$

Std Dev $=0.0029$

$110 \mathrm{mg}$ Ni catalyst, run 1

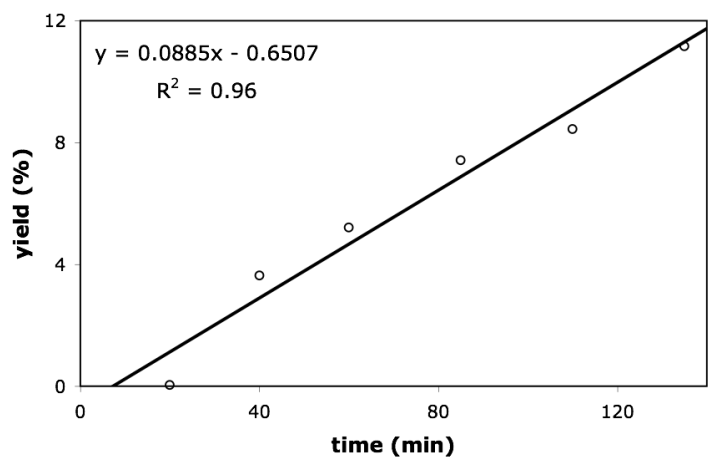

$110 \mathrm{mg}$ Ni catalyst, run 2

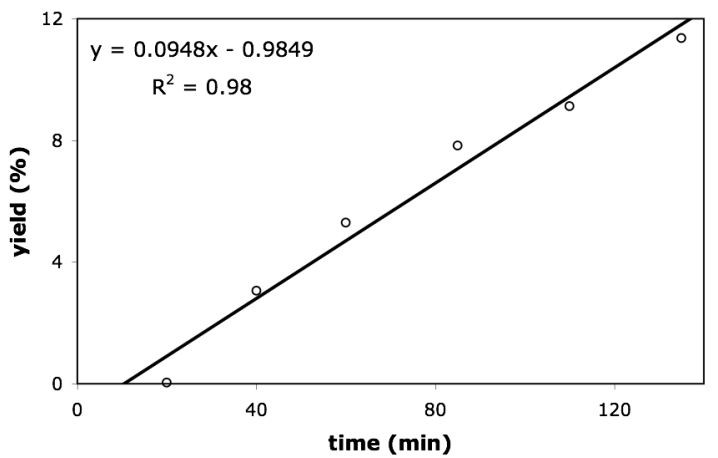

$110 \mathrm{mg}$ Ni catalyst, run 3

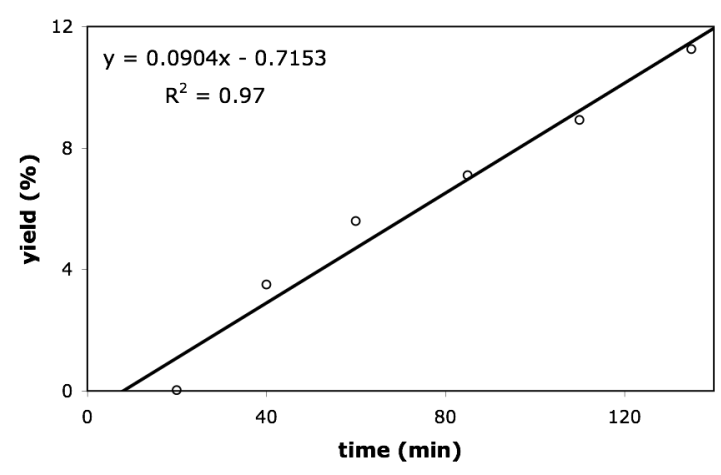


$165 \mathrm{mg}$ Ni catalyst

Rate $=0.1714$

Std Dev $=0.0143$

$165 \mathrm{mg}$ Ni catalyst, run 1

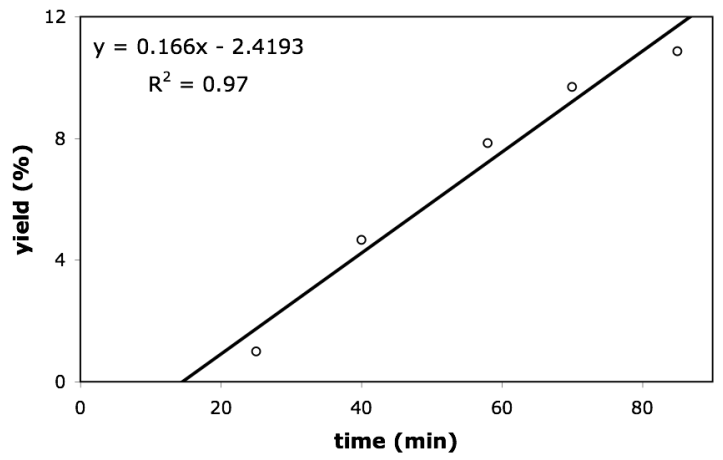

$165 \mathrm{mg}$ Ni catalyst, run 2

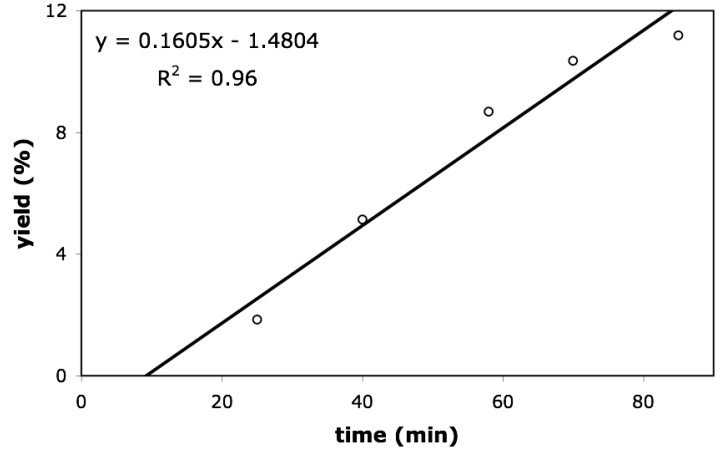

165 mg Ni catalyst, run 3

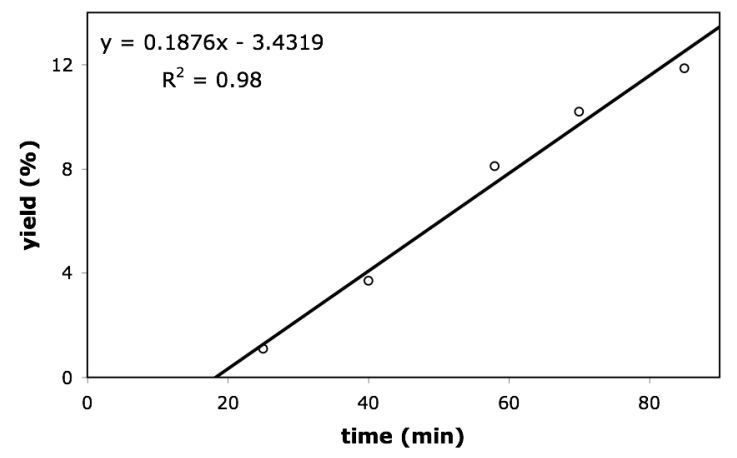


$220 \mathrm{mg}$ Ni catalyst

Rate $=0.1952$

Std Dev $=0.0190$

$220 \mathrm{mg}$ Ni catalyst, run 1

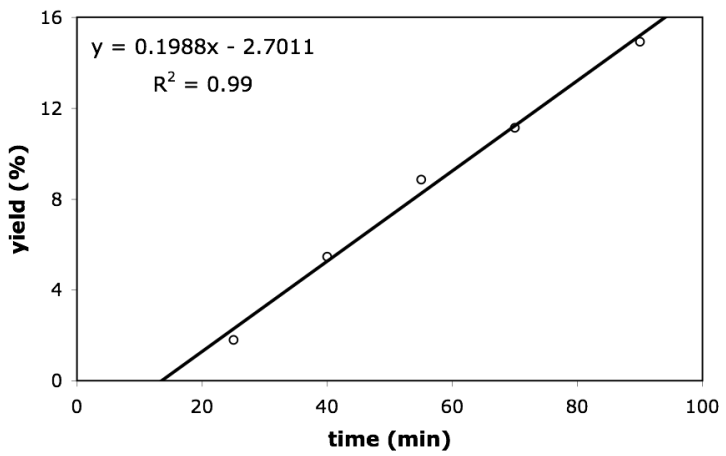

$220 \mathrm{mg}$ Ni catalyst, run 2

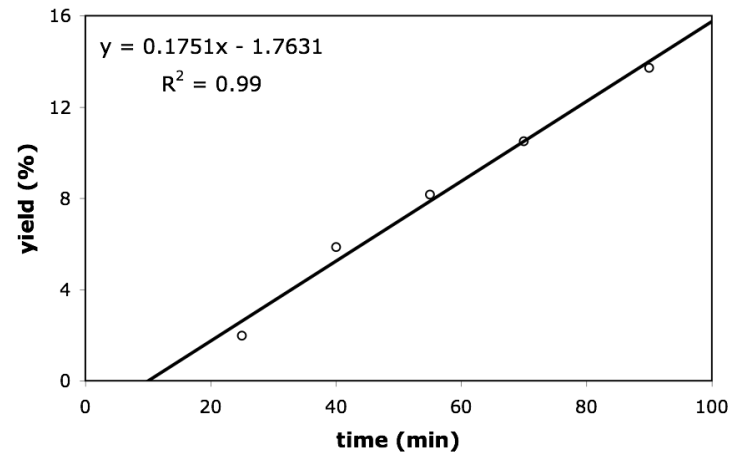

$220 \mathrm{mg}$ Ni catalyst, run 3

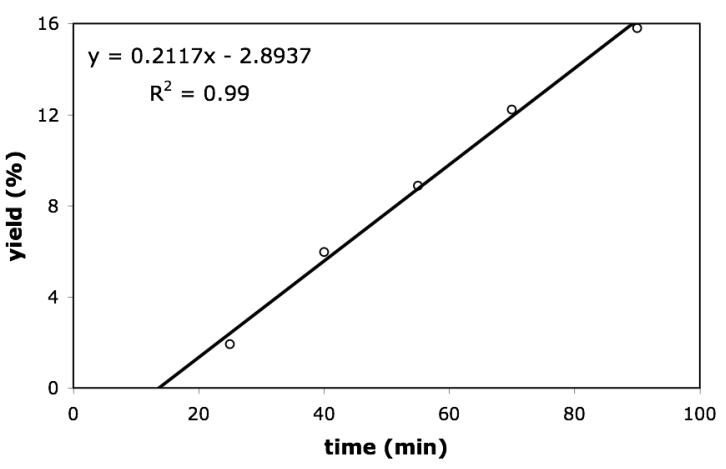




\subsection{Kinetic studies for Michael addition}

The $1^{\circ}$ and $2^{\circ}$ amines of microencapsulated catalyst $\mathbf{1}$ were acylated in the following manner: methanol $(2.5 \mathrm{~mL})$ was added to microcapsule catalyst $(500 \mathrm{mg})$ loaded in a syringe equipped with a frit. Acetic anhydride $(3 \mathrm{~mL})$ was drawn into the syringe and the mixture was rocked at room temperature overnight. The $\mu$ caps were washed with methanol and dried under vacuum before use.

Acylated $\mu$ caps were swollen in $0.5 \mathrm{~mL}$ methanol in a $4 \mathrm{~mL}$ glass vessel before use. Nickel catalyst (2,16.2 mg, $2 \mathrm{~mol} \%$ ), trans- $\beta$-nitrostyrene $(\mathbf{5}, 149.2 \mathrm{mg}, 1 \mathrm{mmol})$, dimethyl malonate $(137 \mu \mathrm{L}, 1.2 \mathrm{mmol})$, and toluene $(0.5 \mathrm{~mL})$ were added to the vessel, which was sealed with a screw cap. The reaction was rocked at $297 \mathrm{~K}$ on a rocker. Reaction conversion was monitored by withdrawing aliquots from the reaction at different time intervals, diluting with methylene chloride, and analyzing by $\mathrm{GC}$ with reference to mesitylene.

Data Summary:

\begin{tabular}{cccc}
\hline mg $\mu$ caps & mole $\%$ urea & Rate $(\mathrm{mmol} \mathrm{7a} / \mathrm{min})$ & std dev \\
\hline 0 & 0 & 0.0101 & 0.0003 \\
5 & 2.82 & 0.0122 & 0.0007 \\
15 & 8.46 & 0.0169 & 0.0008 \\
30 & 16.9 & 0.0231 & 0.0005 \\
\hline
\end{tabular}

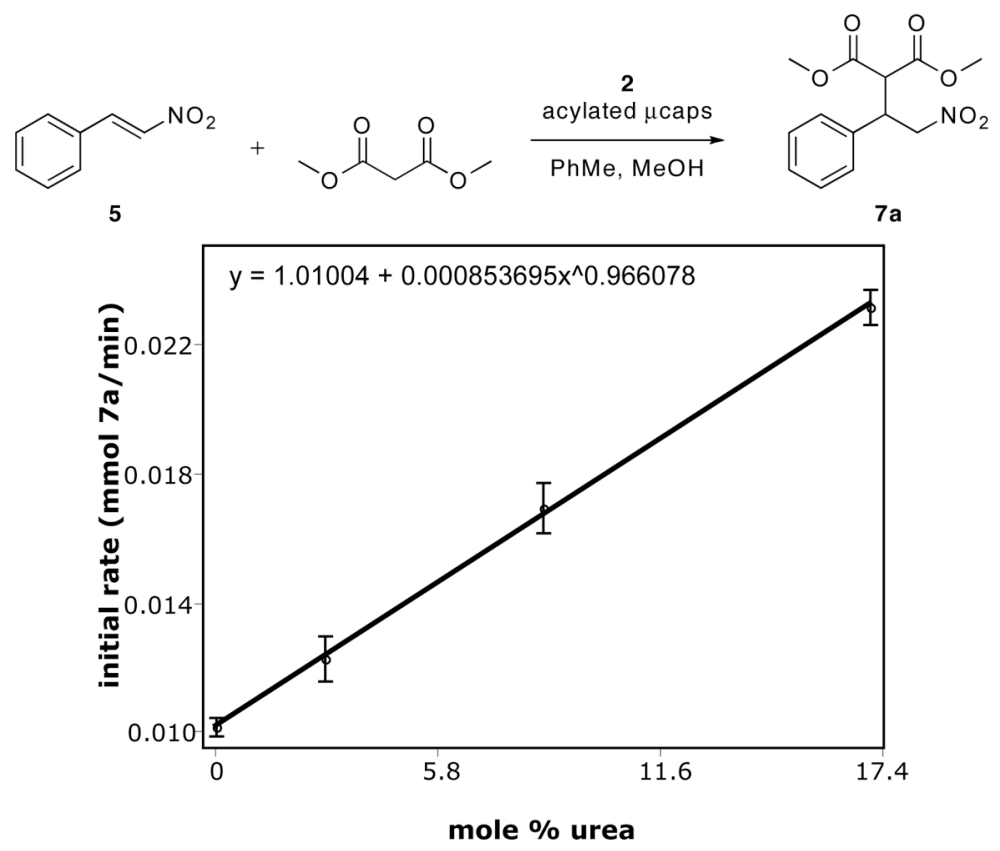


Initial Rate Data:

0 mg $\mu$ caps

Rate $=1.014$

Std Dev $=0.029$

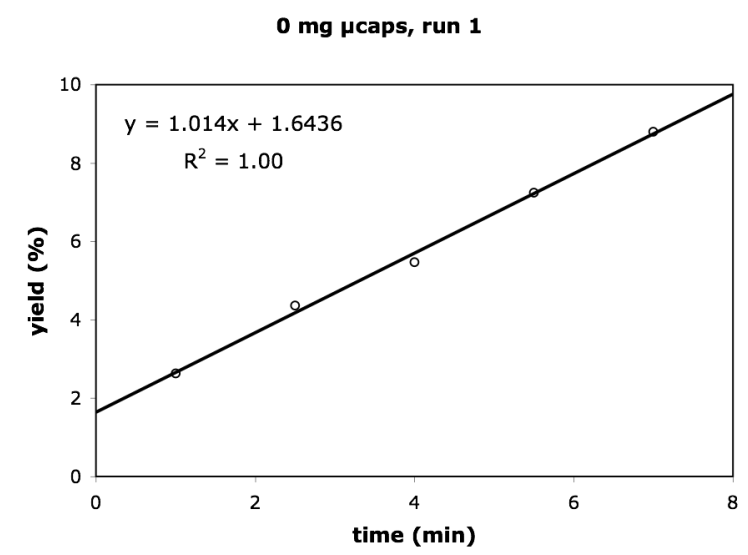

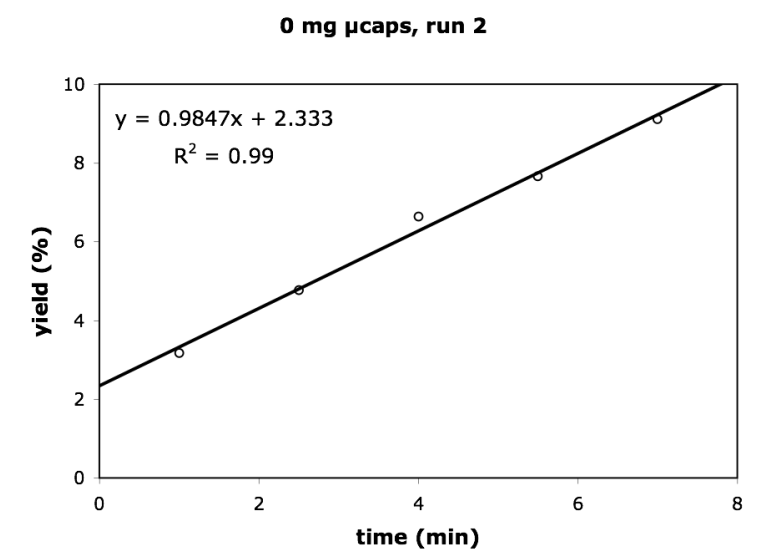

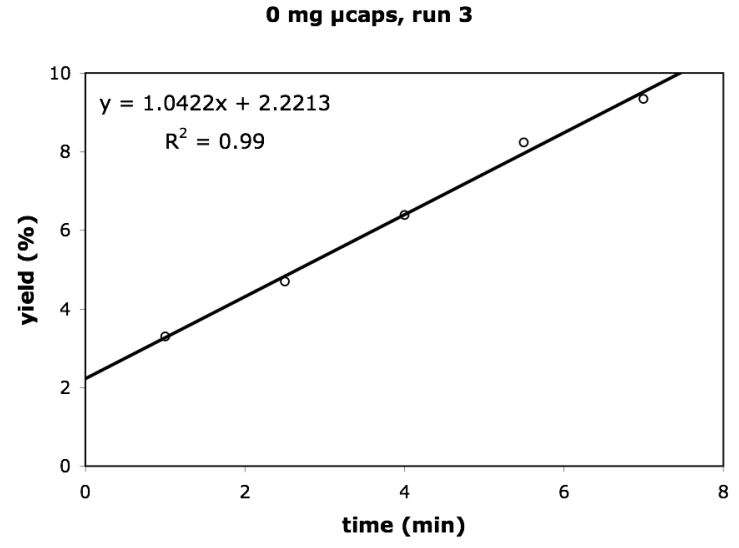


5 mg $\mu$ caps

Rate $=1.224$

Std Dev $=0.070$

5 mg rcaps, run 1

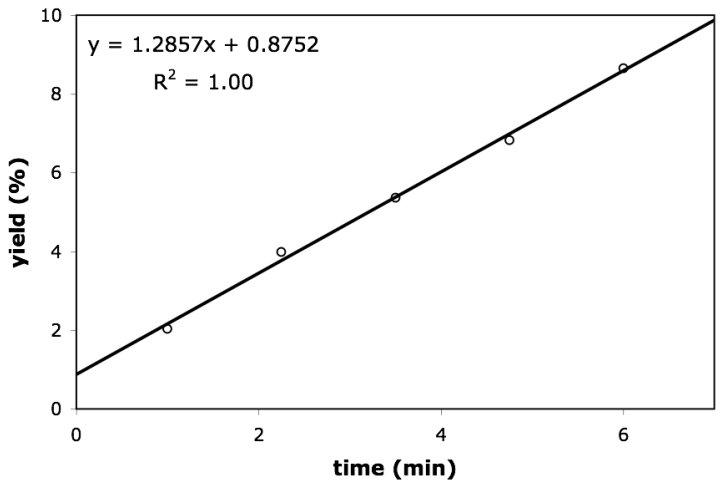

5 mg pcaps, run 2

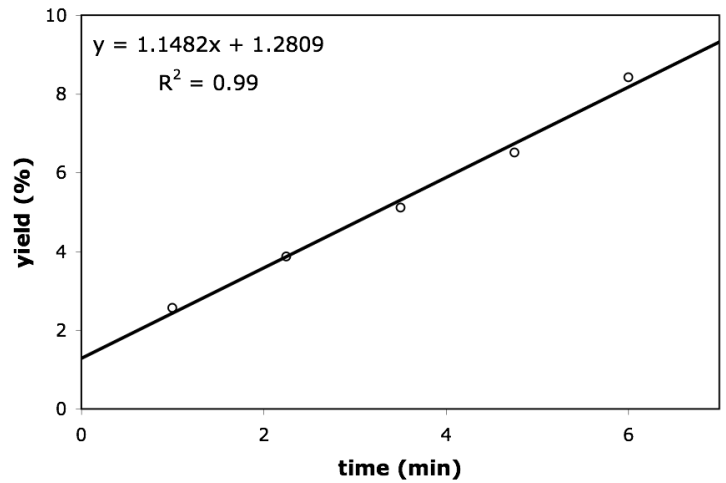

5 mg ucaps, run 3

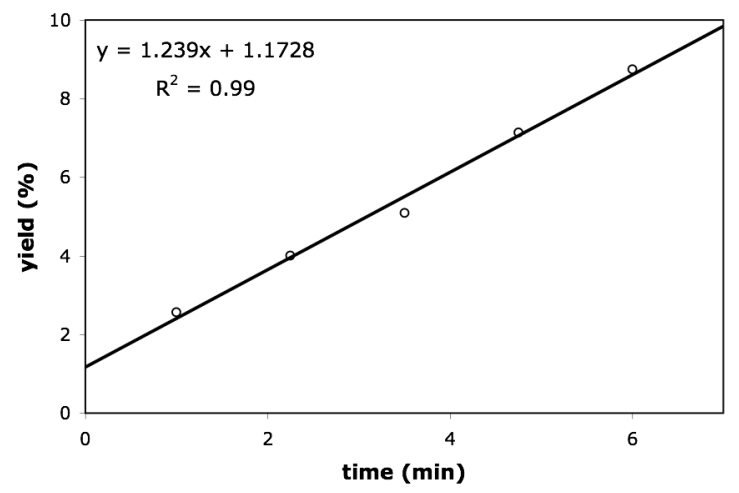


$15 \mathrm{mg} \mu$ caps

Rate $=1.691$

Std Dev $=0.077$

15 mg rcaps, run 1

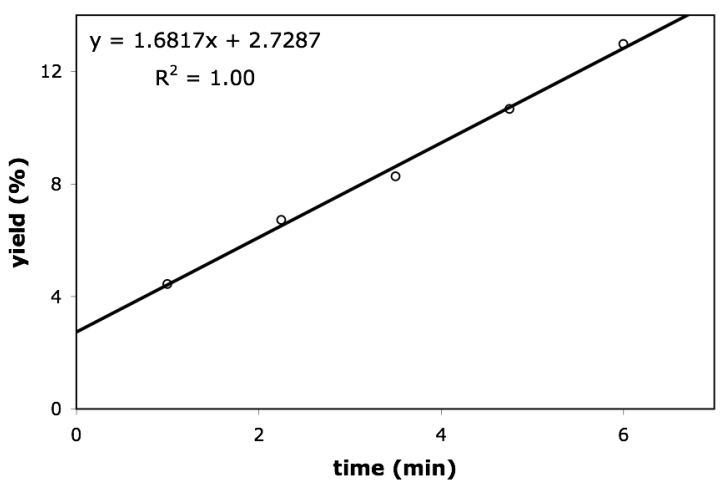

15 mg Hcaps, run 2

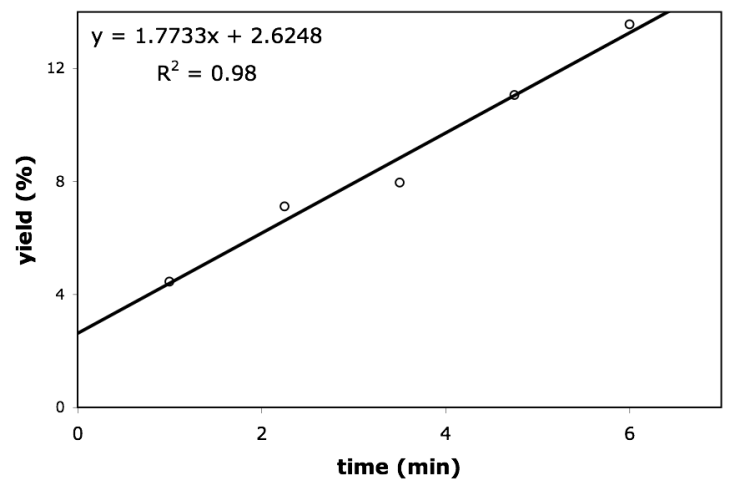

15 mg rcaps, run 3

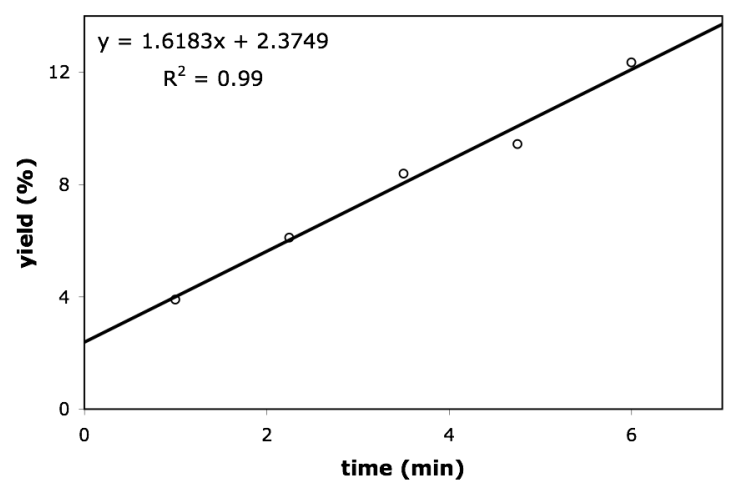


$30 \mathrm{mg} \mu$ caps

Rate $=2.308$

Std Dev $=0.054$

$30 \mathrm{mg}$ Hcaps, run 1

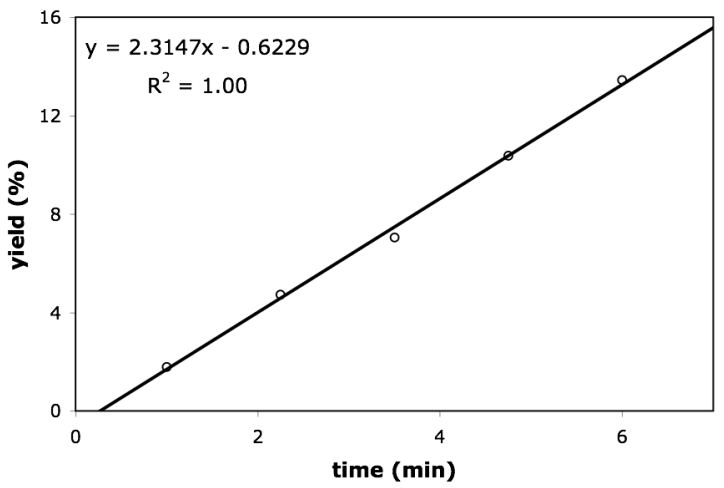

30 mg $\mu$ caps, run 2

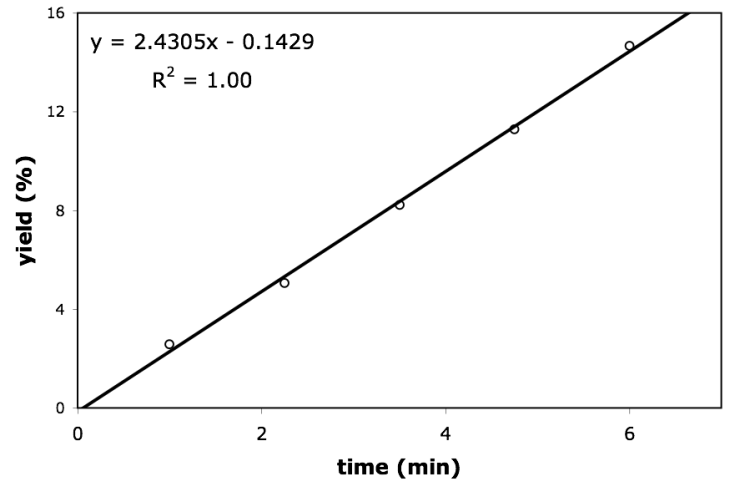

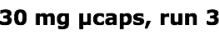

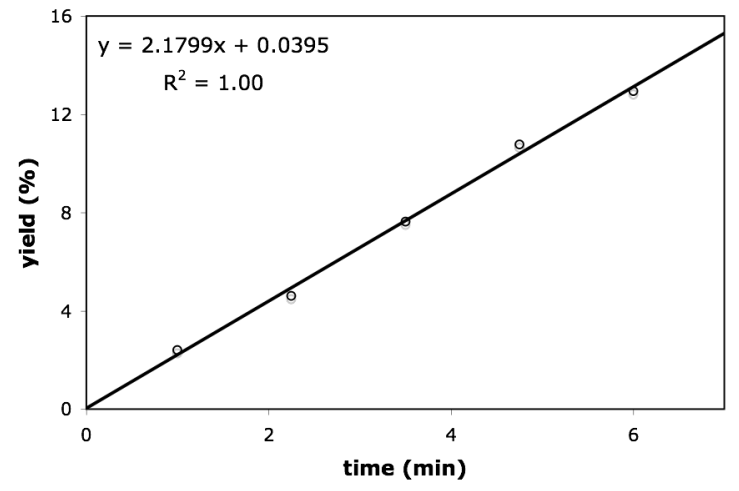




\section{Synthesis of pregabalin (3)}

\subsection{Stepwise synthesis of pregabalin (3)}

(S)-Methyl-2-carbomethoxy-3-(nitromethyl)-4-methylpentanoate ( $S$-7i). Microencapsulated PEI catalyst $(\mathbf{1}, 250 \mathrm{mg}, 11.5 \mathrm{~mol} \%)$ was swollen in $5 \mathrm{~mL}$ methanol in a $50 \mathrm{~mL}$ glass vessel before use. $S$-Nickel catalyst $(S-2,550 \mathrm{mg}, 6.8 \mathrm{~mol} \%), 3$-methylbutyraldehyde $(\mathbf{8}, 1.63 \mathrm{~mL}, 15$ $\mathrm{mmol})$, nitromethane $(5.4 \mathrm{~mL}, 0.1 \mathrm{~mol})$, dimethyl malonate $(1.14 \mathrm{~mL}, 10 \mathrm{mmol})$, and toluene ( 25 $\mathrm{mL}$ ) were added to the vessel, which was sealed with a screw cap. The reaction was rocked at room temperature on a rocker for 48 hours. The $\mu$ caps were filtered off by vacuum filtration. The volatile components were removed under reduced pressure and the product was purified by column chromatography (20\% EtOAc/hexanes) to give $2.46 \mathrm{~g}(94 \%)$ of a colorless oil. Spectral data is given above. Enantiomeric excess was determined by HPLC with a Chiralcel OD-H column (94:6 hexanes:isopropanol, $1.0 \mathrm{~mL} / \mathrm{min} 215 \mathrm{~nm}$; minor enantiomer $t_{r}=6.5 \mathrm{~min}$, major enantiomer $t_{r}=11.3 \mathrm{~min} ; 72 \%$ ee.

Methyl 4-isobutyl-2-oxopyrrolidine-3-carboxylate (9). A solution of ( $\boldsymbol{S})$-7i (1.05 g, 5.26 $\mathrm{mmol}$ ) and $10 \mathrm{~mL}$ EtOH was added to a hydrogenation bottle containing Raney $\mathrm{Ni}(0.85 \mathrm{mg}$, water wet) at room temperature under $\mathrm{N}_{2}$. The flask was evacuated and refilled with hydrogen three times and the reaction was shaken on a Parr hydrogenation apparatus at room temperature and 45 psi for 18 hours. Upon completion, the reaction mixture was filtered through Celite 545 and washed with EtOH. The volatile components were removed under reduced pressure and the product was purified by column chromatography $\left(4 \% \mathrm{MeOH} / \mathrm{CHCl}_{3}\right)$ to give $770 \mathrm{mg}(96 \%)$ of a colorless oil that solidified upon standing. ${ }^{1} \mathrm{HNMR}\left(400 \mathrm{MHz}, \mathrm{CDCl}_{3}\right) \delta 7.6(\mathrm{~s}, 1 \mathrm{H}), 3.72(\mathrm{~s}$, $3 \mathrm{H}), 3.48-3.42(\mathrm{t}, 1 \mathrm{H}), 3.05-3.02(\mathrm{~d}, 1 \mathrm{H}), 2.91-2.86(\mathrm{~m}, 2 \mathrm{H}), 1.50-1.44(\mathrm{~m}, 1 \mathrm{H}), 1.38-1.25(\mathrm{~m}$, $2 \mathrm{H}), 0.83-0.78(\mathrm{~m}, 6 \mathrm{H}) ;{ }^{13} \mathrm{CNMR}\left(400 \mathrm{MHz}, \mathrm{CDCl}_{3}\right) \delta 174.0,170.6,55.0,52.8,47.1,43.3,37.5$, 26.1, 22.7, 22.7 .

(S)-3-Aminomethyl-5-methyl-hexanoic acid (Pregabalin) hydrochloride (3). $9(80 \mathrm{mg}, 0.4$ $\mathrm{mmol})$ and $5 \mathrm{M} \mathrm{HCl}_{\mathrm{aq}}(3 \mathrm{~mL})$ were heated at $125^{\circ} \mathrm{C}$ for 18 hours. Upon cooling, the mixture was extracted with EtOAC $(4 \times 10 \mathrm{~mL})$. The aqueous layer was stirred with activated charcoal at 70 ${ }^{\circ} \mathrm{C}$ and filtered through Celite. The volatile components were removed under reduced pressure to give $75 \mathrm{mg}(95 \%)$ as a white solid. ${ }^{1} \mathrm{HNMR}\left(400 \mathrm{MHz}, \mathrm{CD}_{3} \mathrm{OD}\right) \delta 2.95-2.92(\mathrm{~d}, 2 \mathrm{H}), 2.41-2.37-$ $2.28(\mathrm{~d}, 2 \mathrm{H}), 2.21-2.16(\mathrm{~m}, 1 \mathrm{H}), 1.68-1.61(\mathrm{~m}, 1 \mathrm{H}), 1.24-1.20(\mathrm{t}, 2 \mathrm{H}), 0.96-0.89(\mathrm{dd}, 6 \mathrm{H})$; ${ }^{13} \mathrm{CNMR}\left(400 \mathrm{MHz}, \mathrm{CD}_{3} \mathrm{OD}\right) \delta 175.8,44.5,41.9,37.1,32.5,26.1,23.2,22.4$. For the purposes of analysis, the product was converted to the zwitterion by the addition of $\mathrm{NaOH}$ so that $\mathrm{pH}=$ 7.5-8.5 and then recrystallized from isopropanol/water. The amine was Boc-protected and the acid converted to the methyl amide as follows: Boc-protection was achieved by stirring the zwitterion in the presence of di-tert-butyl dicarbonate, and $\mathrm{NaHCO}_{3}$ in a 1:1 mixture of THF:water. ${ }^{[3]}$ Amide formation was achieved by treatment with methyl amine in the presence of $\mathrm{N}$-methylmorpholine and isobutyl chloroformate in THF. ${ }^{[3]}$ Enantiomeric excess was determined by HPLC with a Chiralcel OD-H column (95:5 hexanes:isopropanol, $0.7 \mathrm{~mL} / \mathrm{min}, 215 \mathrm{~nm}$ ); minor enantiomer $t_{r}=4.4 \mathrm{~min}$, major enantiomer $=7.8 \mathrm{~min} ; 91.5 \%$ ee. 


\subsection{Condensed synthesis of pregabalin (3)}

(S)-Methyl-2-carbomethoxy-3-(nitromethyl)-4-methylpentanoate ( $S$-7i). Microencapsulated PEI catalyst $(\mathbf{1}, 250 \mathrm{mg}, 11.5 \mathrm{~mol} \%)$ was swollen in $5 \mathrm{~mL}$ methanol in a $50 \mathrm{~mL}$ glass vessel before use. $S$-Nickel catalyst $(S-2,550 \mathrm{mg}, 6.8 \mathrm{~mol} \%), 3$-methylbutyraldehyde $(\mathbf{8}, 1.63 \mathrm{~mL}, 15$ $\mathrm{mmol})$, nitromethane $(5.4 \mathrm{~mL}, 0.1 \mathrm{~mol})$, dimethyl malonate $(1.14 \mathrm{~mL}, 10 \mathrm{mmol})$, and toluene ( 25 $\mathrm{mL}$ ) were added to the vessel, which was sealed with a screw cap. The reaction was rocked at room temperature on a rocker for 48 hours. The $\mu$ caps were filtered off by vacuum filtration, 1 $\mathrm{mL}$ PEI was added to the filtrate, and the mixture was stirred until all of the nickel had been chelated, indicated by a color change from green to yellow. The mixture was filtered through celite, the volatile compounds were removed under reduced pressure, and the crude product was carried directly to the next step. The remainder of the synthesis was performed as described above to afford $1.68 \mathrm{~g}(85.7 \%)$ of (S)-3-aminomethyl-5-methyl-hexanoic acid (pregabalin) hydrochloride (3). Spectral data is given above.

References:

[1] Poe, S. L.; Kobašlija, M.; McQuade, D. T. J. Am. Chem. Soc. 2006, 128, 15586-15587.

[2] Evans, D. A.; Seidel, D. J. Am. Chem. Soc. 2005, 127, 9958-9959.

[3] Shendage, D. M.; Fröhlich, R.; Haufe, G. Org. Lett. 2004, 6, 3675-3678. 